\title{
Maxillary Implant-supported Hybrid Prosthesis Fabricated using a Polyetherketoneketone Framework: A Case Report
}

\author{
Ji Hoon Jun, DDS ${ }^{1+}$, Kyung Chul Oh, DDS, PhD ${ }^{2+}$, Hong Seok Moon, DDS, MSD, PhD ${ }^{3 *}$ \\ ${ }^{1}$ Graduate student, Department of Prosthodontics, Yonsei University College of Dentistry, Seoul, Korea \\ ${ }^{2}$ Clinical assistant professor, Department of Prosthodontics, Yonsei University College of Dentistry, Seoul, Korea \\ ${ }^{3}$ Professor, Department of Prosthodontics, Yonsei University College of Dentistry, Seoul, Korea \\ ${ }^{\dagger}$ These authors contributed equally to this work. \\ *Corresponding author: Hong Seok Moon, Department of Prosthodontics, Yonsei University College of \\ Dentistry, 50-1 Yonsei-ro, Seodaemun-gu, Seoul 03722, Korea. \\ Tel: +82-2-2228-3155. Fax:+82-2-312-3598. E-mail: hsm5@yuhs.ac
}

\begin{abstract}
This clinical report describes a case involving a 74-year-old woman with maxillary edentulism who was successfully treated using an implant-supported hybrid prosthesis. The framework of the prosthesis was fabricated using polyetherketoneketone (PEKK), which is a high-performance polymer, and individual lithium disilicate ceramic crowns were cemented for optimal esthetics. No mechanical or biological complications were observed during a 3-year follow-up period. Further clinical studies and long-term clinical data regarding the usefulness of PEKK as a framework material for dental prostheses are necessary.
\end{abstract}

Keywords: Edentulism, Framework, Implant-supported hybrid prosthesis, Polyetherketoneketone

\section{Introduction}

Received: March 5, 2020

Revised: March 22, 2020

Accepted: March 28, 2020

ORCID

Ji Hoon Jun

https://orcid.org/0000-0001-8017-2088

Kyung Chul Oh

https://orcid.org/0000-0003-4584-2597

Hong Seok Moon

https://orcid.org/0000-0001-8118-8145

Copyright $($ C 2020. The Korean Academy of Oral \& Maxillofacial Implantology

An implant-supported hybrid prosthesis is a viable option for the rehabilitation of completely edentulous patients with resorbed alveolar ridges. ${ }^{1}$ It incorporates the features of both removable and fixed prostheses and restores lip support, compensates for oral soft tissue defects, and is functionally and psychologically beneficial for the patient. This type of prosthesis usually consists of a framework and a veneering material. ${ }^{2}$ Conventionally, the framework has been fabricated using titanium, nonprecious or precious metals, or zirconia.

Recently, polyaryletherketones (PAEKs), a group of high-performance thermoplastic polymers, have garnered increased attention as an alternative framework material. Non-Commercial License (http://creativecommo org/licenses/by-nc/4.0/) which permits unrestricted non-commercial use, distribution, and reproduction in any medium, provided the original work is properly Members of this group exhibit excellent chemical stability, low density, high strength, and 
good rigidity over a broad temperature range. Therefore, they are considered suitable materials for use in the airplane and car-manufacturing industries. ${ }^{3}$ In addition, their outstanding biocompatibility has enabled their varied applications in the medical field, such as in the fabrication of artificial joints and spinal implants. ${ }^{4}$

Polyetherketoneketone (PEKK) is a member of the PAEK family that is gaining popularity in the field of dentistry. It exhibits high compressive, flexural, and tensile strengths in addition to a remarkable wear resistance. ${ }^{5}$ Owing to its unique properties, PEKK has been recognized by several clinicians as an appropriate material for the framework fabrication of implant-supported prostheses. ${ }^{4,6,7}$ It can act as a shock-absorber, thus preventing the transfer of excessive stresses to the implant fixtures. ${ }^{4}$ Furthermore, it can be veneered with a wide spectrum of dental materials. ${ }^{5,8-10}$ PEKK is lighter than conventional materials, consequently providing increased patient comfort. ${ }^{4}$ However, few reports have documented the clinical applications of PEKK, possibly because of the short history of clinical usage in the dental field. ${ }^{3,4,6,7}$

The present clinical report documents the prosthetic rehabilitation of a patient with maxillary edentulism using an implant-supported hybrid prosthesis comprising a PEKK framework with individually luted lithium disilicate crowns.

\section{II . Case Report}

A 74-year-old woman presented to the Department of Prosthodontics at the $\bigcirc \bigcirc$ Dental Hospital with multiple oral problems including secondary dental caries and severe mobility in multiple teeth. Thorough clinical and radiographic examinations revealed the presence of generalized chronic moderate periodontitis; dental caries involving the maxillary left canine, left first premolar, and right second premolar; and root remnants of the maxillary right first molar (Fig. 1). Several treatment options were suggested to the patient, who rejected the option of a removable definitive prosthesis. Facial analyses were performed for evaluating the vertical dimension and facial profile, and preliminary impressions were made using irreversible hydrocolloid impression material (Aroma Fine Plus, GC Corp., Tokyo, Japan). Subsequently, a tentative treatment plan, involving rehabilitation of the maxillary arch using an implant-supported hybrid prosthesis exhibiting features of both fixed and removable prostheses, was established. In addition, multiple implant-supported fixed dental prostheses were planned for restoration of the mandibular arch.

Teeth with poor prognosis were extracted, followed by placement of provisional dentures in the 

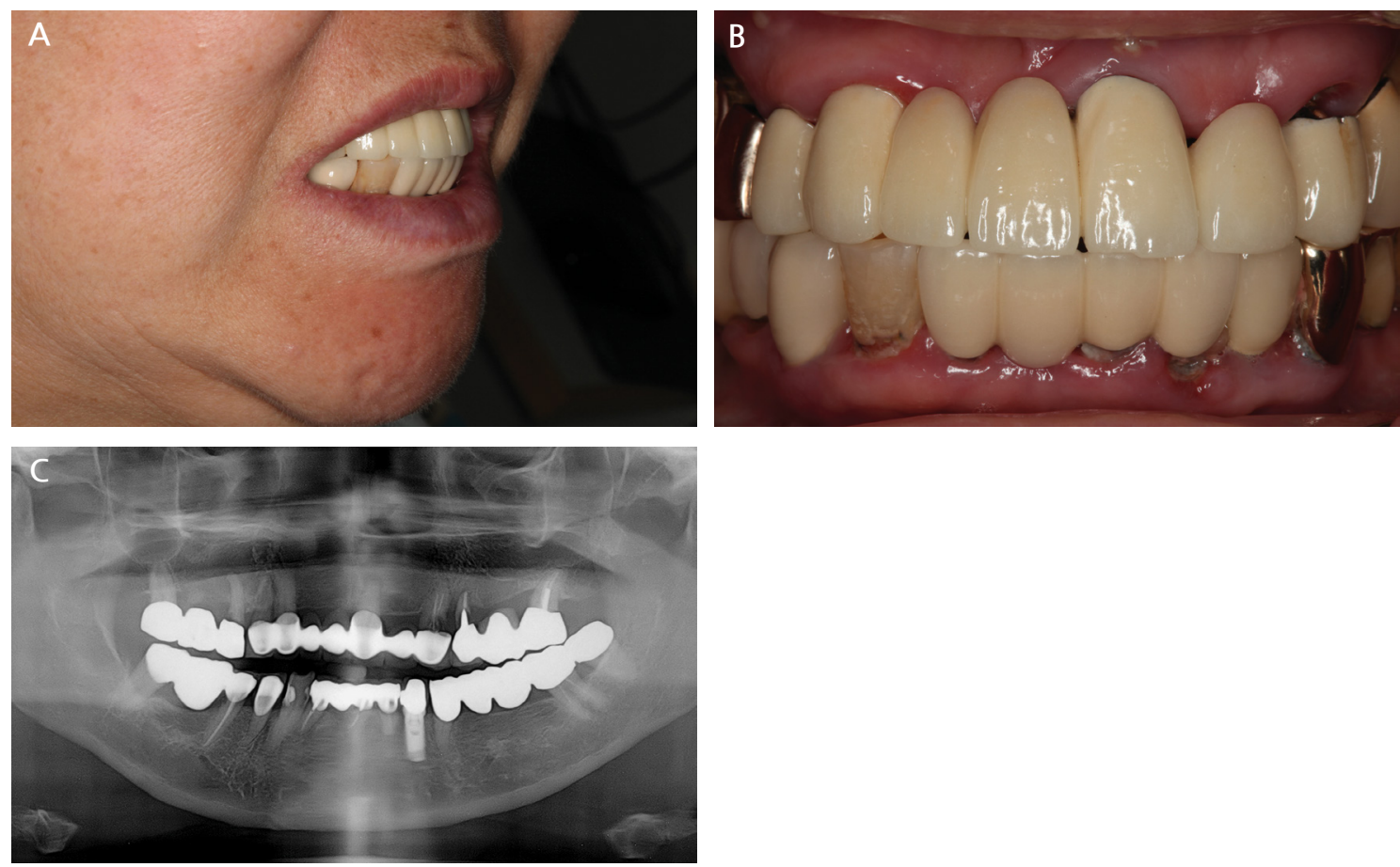

Fig. 1. Preoperative clinical photographs and panoramic radiograph. A, Facial photograph obtained in right lateral view. B, Intraoral photograph obtained in frontal view. C, Panoramic radiograph.

maxilla and mandible. The facial profile and lip support were evaluated, and presence of adequate lip support eliminated the need of a denture flange. A soft liner (Coe-Comfort, GC Corp., Tokyo, Japan) was periodically applied on the provisional dentures during the healing period. After 1 month, preliminary impressions were repeated, followed by registration of the interarch relationship using polyvinylsiloxane bite registration material (O-Bite, DMG Dental, Englewood, NJ) at the adapted vertical dimension.

A diagnostic trial denture using prosthetic teeth (Biotone, Dentsply Sirona, York, PA) was fabricated and tried in the oral cavity for reconfirmation of a satisfactory outcome without a denture flange (Fig. 2). The trial denture was duplicated using polyvinylsiloxane impression material (Aquasil Soft Putty, Dentsply Sirona, York, PA), and radiographic templates were fabricated using autopolymerizing clear acrylic resin (Ortho-Jet, Lang Dental Manufacturing Co., Wheeling, IL). Cone-beam computed tomography was performed with the radiographic templates placed in the patient's mouth, and the following definitive treatment plan was established according to the findings: prosthetic rehabilitation of the maxillary arch using an implant-supported hybrid prosthesis supported by 6 implants and that of the mandibular arch using implant-supported fixed dental prostheses supported by 5 implants. The radiographic templates were modified to function as surgical templates to guide the implant surgery. Implants (Implantium, Dentium, Seoul, Korea) were placed in the bilateral lateral incisor, first premolar, and first molar regions of the maxilla. 
Six months after implant placement, a final impression of the mandibular arch was made using polyether impression material (Impregum Penta, 3M ESPE, Minneapolis, MN). After another 6 months, an implant-level final impression of the maxillary arch was made. The impression copings were splinted using autopolymerizing acrylic resin (GC Pattern Resin, GC Corp., Tokyo, Japan), and a definitive cast was prepared using type IV dental stone (Snowrock, DK Mungyo, Gimhae, Korea). Accurate transfer of the implant fixtures in the oral cavity to the implant analogs on the definitive cast was verified using a set of jigs fabricated from autopolymerizing acrylic resin. ${ }^{11}$

The vertical dimension was determined with reference to physiologic rest position, phonetics and the provisional denture. The centric relation was registered using polyvinylsiloxane using a bimanual
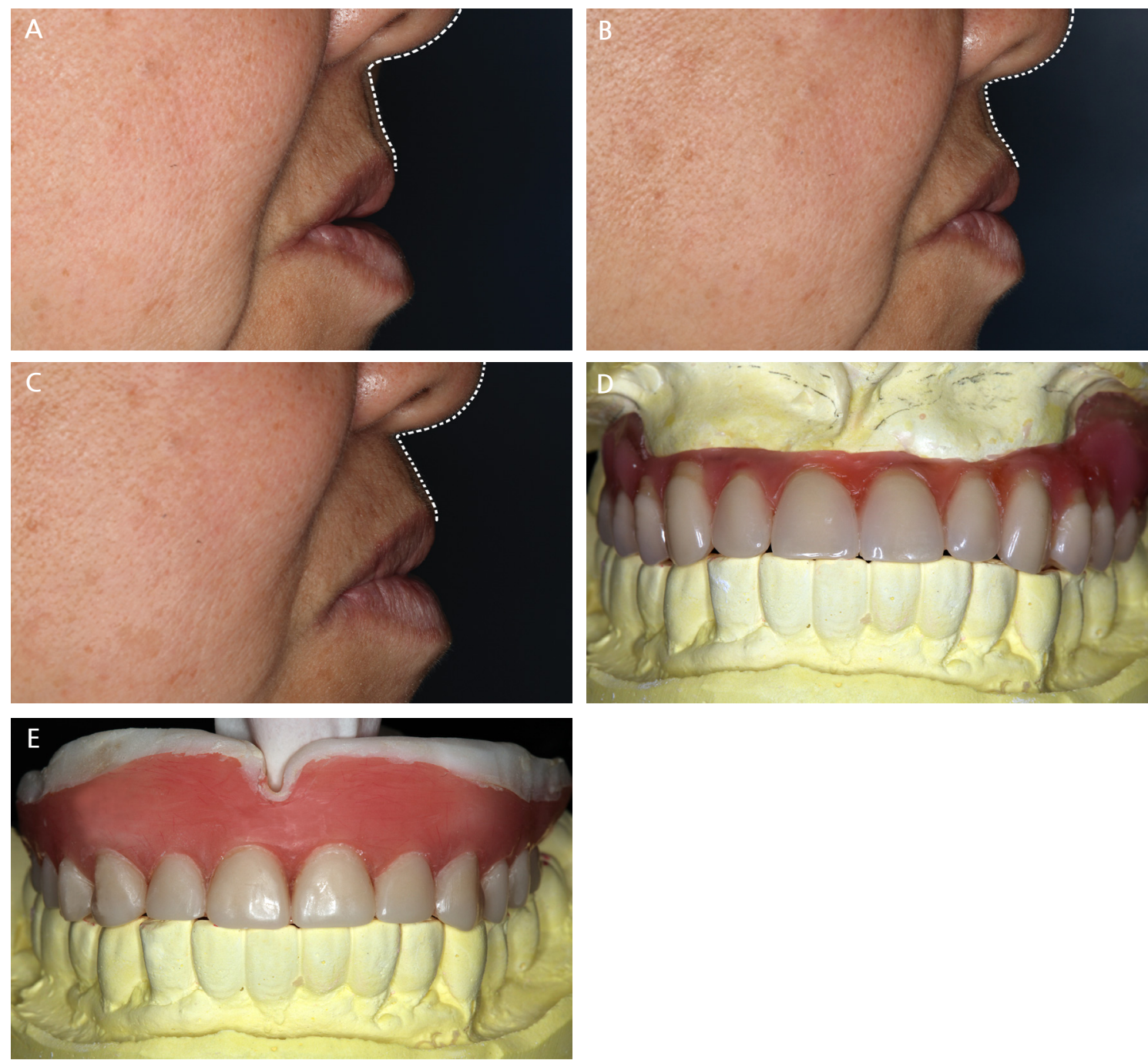

Fig. 2. Clinical evaluation of diagnostic trial denture from the lateral aspect. The dotted lines represent lip support. A, Facial appearance before insertion of the diagnostic trial denture. B, Facial appearance after insertion of the diagnostic trial denture. C, Facial appearance after insertion of the provisional denture. D, Frontal view of diagnostic trial denture without labial flange. E. Frontal view of the provisional denture. 
method, and the definitive cast was mounted on a semiadjustable articulator (Hanau modular articulator, Whip Mix, Louisville, KY). Subsequently, denture teeth were arranged on the maxillary record base and diagnostic wax-ups were prepared for the mandibular teeth. They were placed in the oral cavity and assessed for esthetics and occlusion (Fig. 3). The interarch relationship was additionally verified. Then, implant-supported metal-ceramic fixed dental prostheses were fabricated for the mandibular arch and cemented using methacrylate cement (Premier Implant Cement, Premier Dental Products Co., Plymouth Meeting, PA).

The trial arrangement was scanned using a desktop scanner (Identica Hybrid, Medit Co., Seoul, Korea) for designing the definitive prostheses using a computer-aided designing software program (exocad DentalCAD, exocad GmbH, Daramstadt, Germany). Customized titanium abutments were tried in the patient's mouth, and accurate transfer from the definitive cast to the maxillary arch of the patient was confirmed using a verification jig (Fig. 4). The PEKK (Pekkton Ivory, Cendres+Métaux, Biel/Bienne, Switzerland) framework, which included 14 abutment tooth forms, was milled in a milling machine (Trione Z, DIO Implants, Busan, Korea). The framework was designed in one piece without palatal coverage.
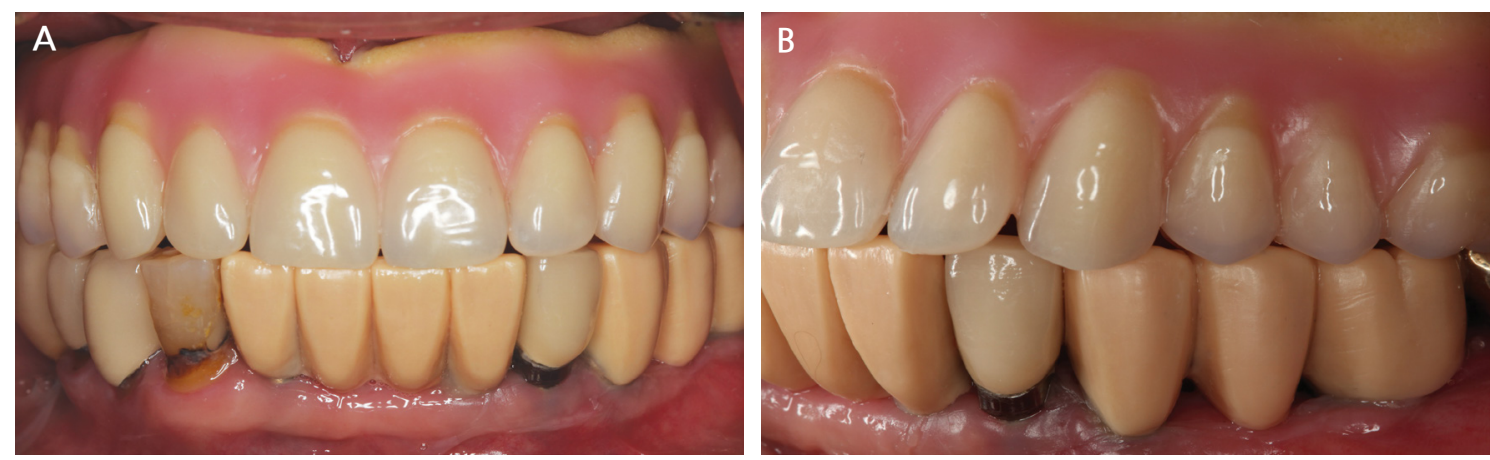

Fig. 3. Clinical evaluation of full-contour trial teeth arrangement. A, Frontal view. B, Lateral view.
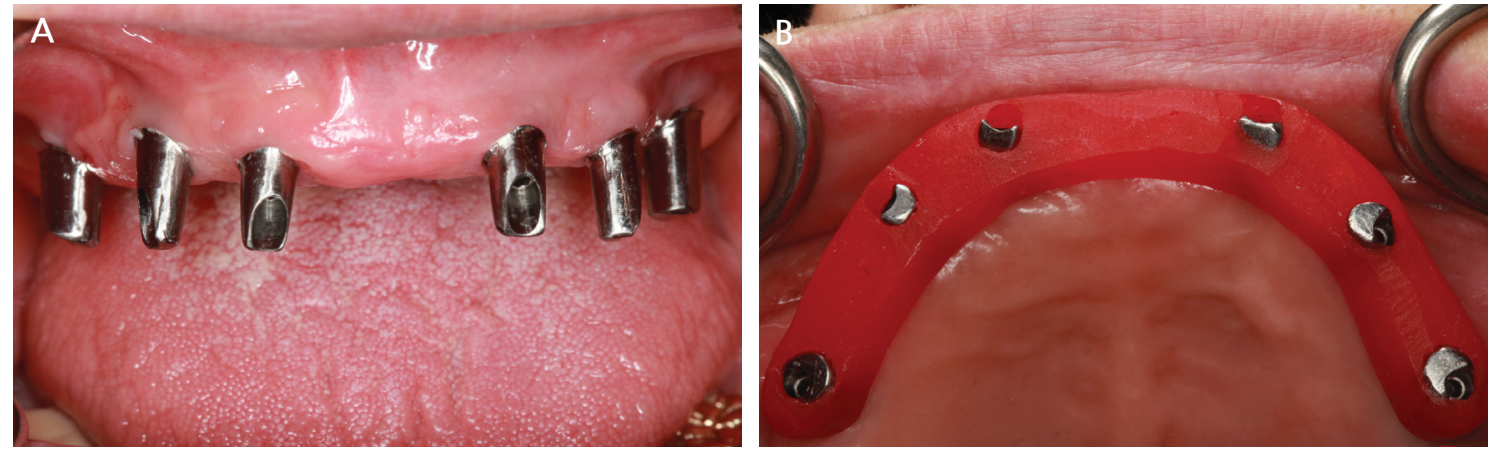

Fig. 4. Verification procedure for maxillary abutments. A, Placement of customized titanium abutments. $B$, Placement of verification jig on titanium abutments. 
A passive fit and favorable adaptation of the framework on the abutments in the patient's mouth were verified with the use of powder spray (Occlude, Pascal, Bellevue, WA) and by alternatively applying figure pressure to the terminal ends of the framework (Fig. 5). The interocclusal relationship was registered using polyvinylsiloxane. Individual lithium disilicate ceramic crowns (IPS e.max CAD, Ivoclar Vivadent, Schaan, Liechtenstein) were designed, milled, and temporarily cemented to the framework using zinc oxide noneugenol cement (Temp-Bond NE, Kerr, Karlsruhe, Germany).

The framework and the temporarily cemented crowns were tried in the oral cavity, and esthetics and
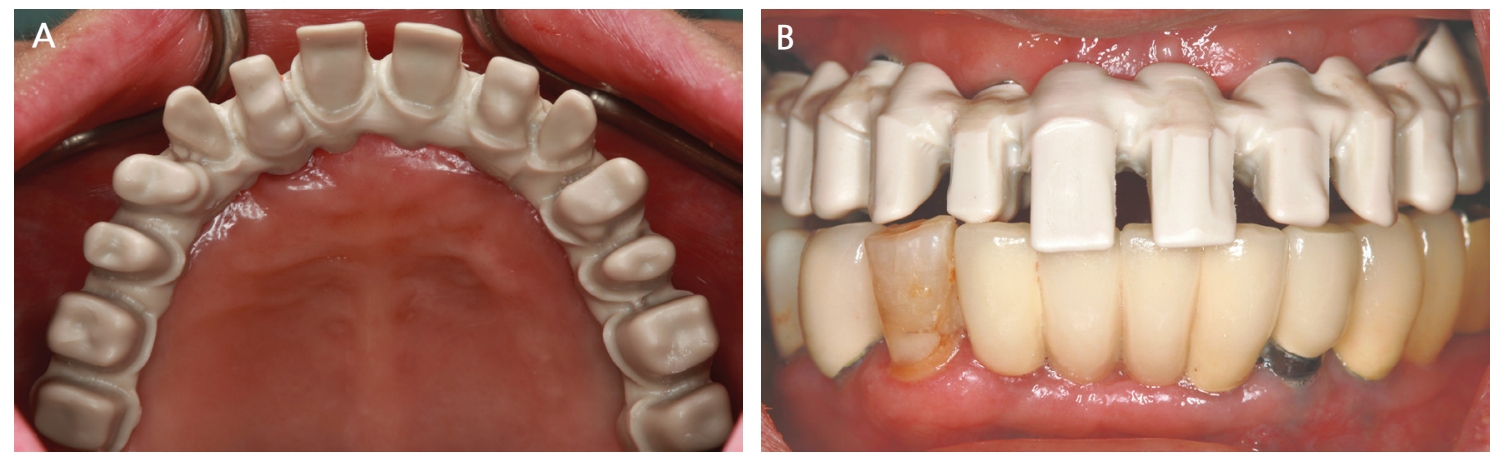

Fig. 5. Intraoral placement of polyetherketoneketone (Pekkton Ivory; Cendres+Métaux) framework. A, Occlusal view. B, Frontal view.
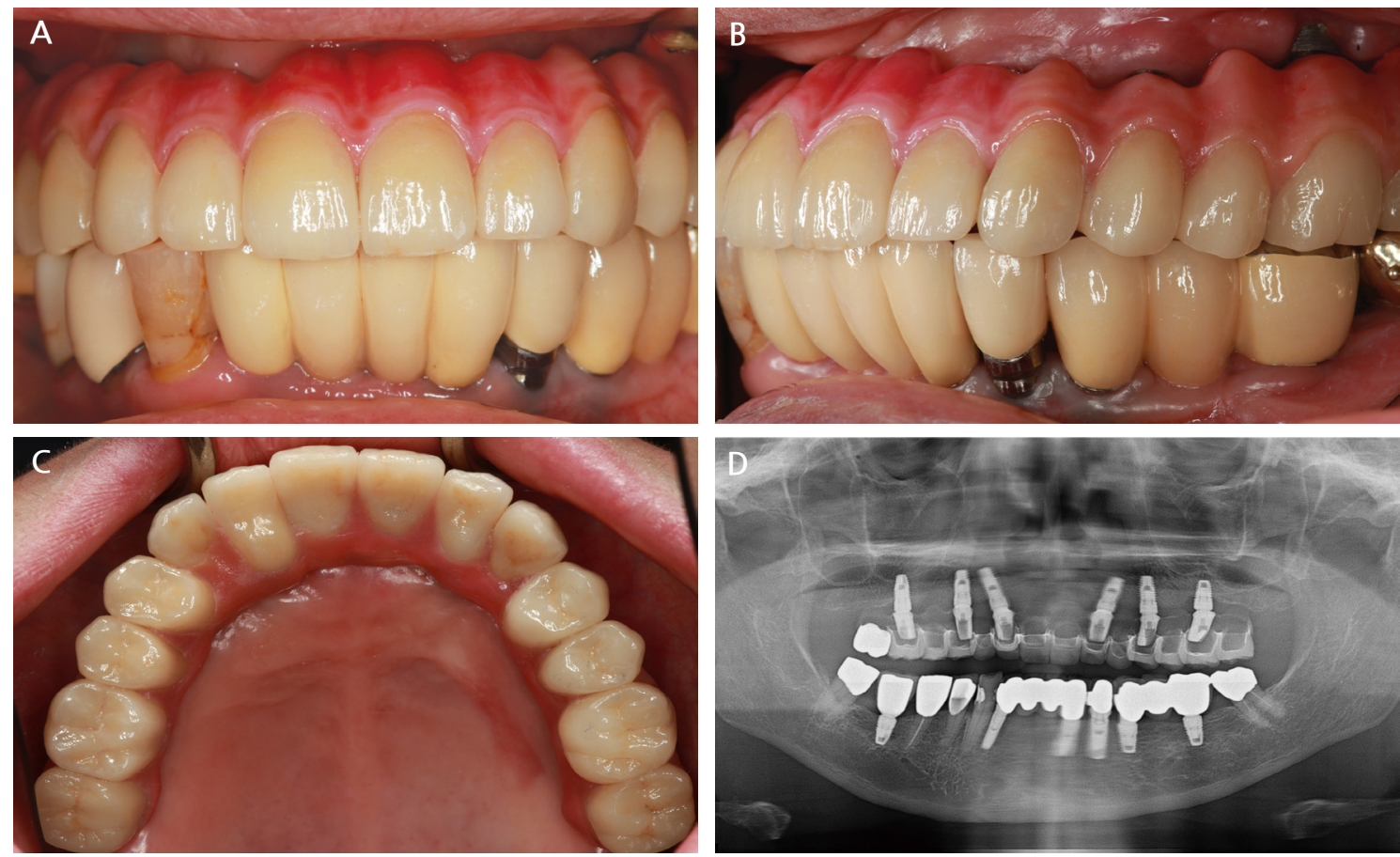

Fig. 6. Postoperative clinical photographs and radiograph. A, Intraoral photograph obtained in frontal view. B, Intraoral photograph obtained in left lateral view. C, Intraoral photograph obtained in occlusal view. D, Panoramic radiograph. 
occlusion were verified. After necessary adjustments, the interarch relationship was registered using polyvinylsiloxane. A gingival opaquer (SR Nexco Opaquer, Ivoclar Vivadent, Schaan, Liechtenstein) and a gingiva-colored composite resin (SR Nexco Paste Gingiva, Ivoclar Vivadent, Schaan, Liechtenstein) were applied to the PEKK framework to match the gingival color and texture.

Once the ceramic crowns were characterized, glazed, and luted to the PEKK framework using resin cement (Multilink, Ivoclar Vivadent, Schaan, Liechtenstein), the definitive maxillary prosthesis was cemented to the titanium abutments using methacrylate cement (Fig. 6). ${ }^{12-14}$ A mutually protected occlusal scheme with shallow anterior guidance was achieved. ${ }^{15}$

Six months after prosthesis delivery, the ceramic crown of the maxillary right second molar was replaced with a zirconia crown for occlusal plane correction. There were no signs or symptoms of mechanical/biological complications during a 3-year follow-up period. The patient was satisfied with the functional and esthetic outcomes.

\section{Discussion}

The PEKK prosthesis, being a fixed type, was able to eliminate palatal coverage for increased convenience and better patient acceptance. A consensus has not been reached on the optimum number of implants for full-arch prostheses. Brånemark suggested utilizing six implants for the maxilla to support a full-arch fixed prosthesis. ${ }^{16}$ According to Bhering et al., all-on-six approach exhibited more favorable biomechanical behavior than all-on-four concept when restoring the edentulous maxilla with one-piece implant prosthesis. ${ }^{17}$ Lambert et al. noted that for the edentulous maxilla, placing more than $\geq 6$ implants resulted in higher prosthodontic survival rates than placing $<6$ implants. ${ }^{18}$ Because six implants supported the framework in this case, the prosthesis could be fabricated to be a fixed-detachable hybrid type with relatively lower risk of prosthodontic failure. Distal cantilevers on both sides were carefully designed and adjusted to avoid implant overloading. ${ }^{19}$ Occlusal contacts on working/ nonworking sides were eliminated from cantilevers on both sides, and their maximal mesiodistal lengths were limited to $10 \mathrm{~mm}$. Moreover, infraocclusion was intentionally established in both the distal cantilever regions for the prevention of excessive occlusal stresses. The cantilevers were subjected to regular occlusal examinations and adjustments.

The mutually protected occlusal scheme was achieved, which has been widely recommended for fullarch implant-supported fixed prostheses opposing a natural dentition. ${ }^{15}$ In maximum intercuspation, contact between the posterior teeth on both sides occurred simultaneously. During right-lateral 
movements, canine-guided occlusion was achieved, whereas during left-lateral movements, group function occlusion was established. This was due to the differing conditions of the mandibular canines. The mandibular right canine exhibited good periodontal health; therefore, canine guidance was designed for maximum esthetics and neuromuscular protection. The maxillary prosthesis was completely splinted; therefore, the anterior and posterior segments were not independent. It was presumed that canine guidance would aid in the even load distribution to the implants. On the left side, the mandibular canine was supported by a single implant. Accordingly, group function was established because a single excursive contact could increase the risk of mechanical and biological implant-related complications. The mandibular incisors were restored using a four-unit implant-supported fixed dental prosthesis, and small horizontal and vertical overlaps were established in the incisor region for the prevention of excessive stresses during protrusion.

Several schools of thought exist regarding the use of cross-arch splinting. Cross -arch stabilization in implant-supported prosthesis offers increased resistance against lateral forces induced by functional and parafunctional movements. ${ }^{19}$ In addition, it evenly distributes the occlusal loads, thus preventing overloading of individual implants; reduces the number of implants required; and minimizes mechanical complications. ${ }^{20}$ However, a passive fit is difficult to achieve in a single-piece prosthesis than in segmented prostheses. Indeed, the verification procedure was repeated several times to achieve an adequate passive fit. The use of verification jigs helped in achieving a clinically passive fit of the prosthesis framework. ${ }^{11}$ Clinicians should identify situations where a segmented PEKK prosthesis may be more practical than a single-piece prosthesis, considering the simple laboratory procedures, ease of achievement of a passive fit, and reduced chair time of segmented prostheses.

The framework was cemented to the abutments rather than screwed to the implants. The advantages and disadvantages of cement-retained and screw-retained implant prostheses have been discussed for a long time, and the better type of the two is still controversial. ${ }^{14}$ Cement-retention was preferred in this case for several reasons. A passive fit of the framework is more likely to be achieved, which prevents the transfer of excessive stresses to the bone and the implant-abutment structure. ${ }^{12-14}$ Further, cementretention can compensate for the varying inclinations of the multiple implants. ${ }^{13,14}$ A provisional resin cement was selected to ensure restoration retrievability, should biological or mechanical complications arise.

The bond strength between PEKK and veneering materials remains a controversial issue. PEKK is opaque and whitish-gray in color, which necessitates the use of an esthetic veneering material that can mimic the soft tissues. Because of the low surface energy of PEKK, it is difficult to achieve a strong 
interfacial bond with composite resins. ${ }^{4}$ Several surface treatment methods have been proposed to overcome this limitation. ${ }^{5,89}$ In accordance with the manufacturer's recommendation, the framework for the present case was roughened by sandblasting with aluminum-oxide particles, followed by the application of a silane coupling agent and a composite primer, and build-up of a composite resin was completed. During the 3-year follow-up period, neither chipping nor delamination of the veneering resin was observed. These findings suggest that PEKK can outclass the conventional materials used for fabrication of prosthesis frameworks, provided a consensus regarding the surface treatment method is established through further clinical studies.

Instead of using acrylic resin denture teeth, individual lithium disilicate ceramic crowns were selected because of their superior esthetics, ease of repair in the event of fracture, and wear resistance. ${ }^{6}$ PEKK, being metal free, was selected because it does not lead to problems of metal allergies, metallic taste, or electrical conductivity (galvanism), commonly associated with conventional metallic framework materials. In addition, because of its low modulus of elasticity, PEKK is expected to show shockabsorbing properties, thus preventing mechanical and biological complications. ${ }^{4}$ Since computer-aided designing/computer-aided manufacturing technology was used for framework fabrication using PEKK, the design could be efficiently adjusted according to the underlying arch anatomy. Furthermore, the milling procedure eliminated the errors associated with casting, such as porosities, gas inclusions, and incomplete casting, thus improving the quality of the prosthesis. Finally, workload and working time of the technicians are considerably lesser with this material than with conventional materials.

Some authors have experimentally showed that the fracture load of a three-unit fixed prosthesis fabricated using PEKK veneered with lithium disilicate was $1526.56 \mathrm{~N}$; these values were $1069.54 \mathrm{~N}$ and $2468.82 \mathrm{~N}$ for PEKK veneered with composite resin and monolithic zirconia, respectively. ${ }^{10}$ Further studies should evaluate the longevity of full-arch prostheses incorporating PEKK frameworks. Careful periodic examinations are mandatory in patients receiving such prostheses because of the current scarcity of long-term clinical data.

\section{Conclusion}

In summary, an elderly woman with maxillary edentulism was successfully treated using an implantsupported, full-arch hybrid prosthesis comprising a PEKK framework with individually cemented lithium disilicate ceramic crowns. The findings from this case suggest that PEKK is a viable option that can replace conventional prosthetic framework materials because of its numerous advantages. Periodic 
examinations of patients receiving these prostheses are necessary until long-term clinical data evaluating PEKK frameworks become available.

\section{References}

1. Egilmez F, Ergun G, Cekic-Nagas I, Bozkaya S. Implant-supported hybrid prosthesis: conventional treatment method for borderline cases. Eur J Dent 2015;9:442-8.

2. Simon H, Yanase RT. Terminology for implant prostheses. Int J Oral Maxillofac Implants 2003;18: 539-43.

3. Klur T, Hasan I, Ottersbach K, Stark H, Fichte M, Dirk C, et al. PEKK-made indirect temporary crowns and bridges: a clinical pilot study. Clin Oral Investig 2019;23:771-7.

4. Han KH, Lee JY, Shin SW. Implant- and tooth-supported fixed prostheses using a high-performance polymer (Pekkton) framework. Int J Prosthodont 2016;29:451-4.

5. Fuhrmann G, Steiner M, Freitag-WolfS, Kern M. Resin bonding to three types of polyaryletherketones (PAEKs)-durability and influence of surface conditioning. Dent Mater 2014;30:357-63.

6. Dawson JH, Hyde B, Hurst M, Harris BT, Lin WS. Polyetherketoneketone (PEKK), a framework material for complete fixed and removable dental prostheses: a clinical report. J Prosthet Dent 2018; 119:867-72.

7. Oh KC, Park JH, Lee JH, Moon HS. Treatment of a mandibular discontinuity defect by using a fibula free flap and an implant-supported fixed complete denture fabricated with a PEKK framework: a clinical report. J Prosthet Dent 2018;119:1021-4.

8. Stawarczyk B, Silla M, Roos M, Eichberger M, Lümkemann N. Bonding behaviour of polyetherketoneketone to methylmethacrylate- and dimethacrylate-based polymers. J Adhes Dent 2017;19:331-8.

9. Fokas G, Guo CY, Tsoi JKH. The effects of surface treatments on tensile bond strength of polyetherketone-ketone (PEKK) to veneering resin. J Mech Behav Biomed Mater 2019;93:1-8.

10. Amelya A, Kim JE, Woo CW, Otgonbold J, Lee KW. Load-bearing capacity of posterior CAD/ CAM implant-supported fixed partial dentures fabricated with different esthetic materials. Int $\mathrm{J}$ Prosthodont 2019;32:201-4.

11. Ercoli C, Geminiani A, Feng C, Lee HJ. The influence of verification jig on framework fit for nonsegmented fixed implant-supported complete denture. Clin Implant Dent Relat Res 2012;14 Suppl 1: e188-95.

12. Michalakis KX, Hirayama H, Garefis PD. Cement-retained versus screw-retained implant restorations: a critical review. Int J Oral Maxillofac Implants 2003;18:719-28.

13. Shadid R, Sadaqa N. A comparison between screw- and cement-retained implant prostheses. A literature review. J Oral Implantol 2012;38:298-307.

14. Wittneben JG, Joda T, Weber HP, Brägger U. Screw retained vs. cement retained implant-supported fixed dental prosthesis. Periodontol. 2000 2017;73:141-51.

15. Kim Y, Oh TJ, Misch CE, Wang HL. Occlusal considerations in implant therapy: clinical guidelines with biomechanical rationale. Clin Oral Implants Res 2005;16:26-35.

16. Daudt PW, Aghaloo T, Emmett TW, Taylor TD, Morton D. (2018). Number of implants placed for complete-arch fixed prostheses: A systematic review and meta-analysis. Clin Oral Implants Res 2018;29(S16):154-83. 
17. Bhering CLB, Mesquita MF, Kemmoku DT, Noritomi PY, Consani RL, Barao VA. Comparison between all-on-four and all-on-six treatment concepts and framework material on stress distribution in atrophic maxilla: A prototyping guided 3D-FEA study. Mater Sci Eng C Mater Biol Appl 2016;69:715-25.

18. Lambert FE, Weber HP, Susarla SM, Belser UC, Gallucci GO. Descriptive analysis of implant and prosthodontic survival rates with fixed implant-supported rehabilitations in the edentulous maxilla. J Periodontol 2009;80(8):1220-30.

19. Gross MD. Occlusion in implant dentistry. A review of the literature of prosthetic determinants and current concepts. Aust Dent J 2008;53 Suppl 1: S60-8.

20. Abduo J, Bennani V, Waddell N, Lyons K, Swain M. Assessing the fit of implant fixed prostheses: a critical review. Int J Oral Maxillofac Implants 2010;25:506-51. 